\title{
Image-Guided Analysis of Shoulder Pathologies: Modelling the 3D Deformation of the Subacromial Space during Arm Flexion and Abduction
}

\author{
Alexandra Branzan Albu ${ }^{1}$, Denis Laurendeau ${ }^{1}$, Luc. J. Hébert ${ }^{2}$, Hélène Moffet ${ }^{3}$, \\ Marie Dufour $^{2,4}$, and Christian Moisan ${ }^{2,4}$ \\ ${ }^{1}$ Computer Vision and Systems Laboratory, Department of Electrical and Computer \\ Engineering, Laval University, Québec (Qc) G1K 7P4, Canada \\ $\{$ Branzan, Laurend\} @gel.ulaval.ca \\ 2 Department of Radiology, Laval University, Québec(Qc), G1K7P4, Canada \\ ${ }^{3}$ Department of Rehabilitation, Laval University, Québec(Qc), G1K7P4, Canada \\ ${ }^{4}$ IMRI Unit, Québec City University Hospital, Québec (Qc) G1L 3L5, Canada
}

\begin{abstract}
This paper describes a simple, yet efficient method for modelling complex musculo-skeletal structures in motion. The proposed approach contains three main modules: segmentation, 3D reconstruction, and feature extraction. The segmentation module integrates region and edge information in a coherent manner. The 3D reconstruction technique is based on morphological morphing between adjacent slices and on contour-based extrapolation of extreme slices. Model validation is a rather challenging task, since no direct access is possible to in vivo human bony structures of the shoulder complex. We implement an internal validation approach, based on comparing the three models built for the same human shoulder. Finally, two descriptors of the subacromial space deformation during arm motion are computed. Their reliability is assessed using statistics of the healthy human shoulder.
\end{abstract}

\section{Introduction}

The painful shoulder is one of the most common musculo-skeletal complaints, and without an accurate diagnosis and treatment it may result in functional loss and disability in the patients that it affects. The successful evaluation of shoulder pain is rather complex and it relies on a hierarchical scheme for the differential diagnosis of many disorders [1]. Motion plays an important role in the evaluation process, which is either performed directly by the physical therapist or based on medical imaging techniques. Thus, physical examination is primarily based on the assessment of the active range of motion (ROM), and uses combinations of four basic types of shoulder movements: abduction, flexion, internal rotation and external rotation. While standard protocols of clinical evaluation perform well in diagnosing disorders such as adhesive 
capsulitis and shoulder instability, magnetic resonance examinations are used for the diagnosis of the shoulder impingement syndrome and of the rotator cuff disease.

The recent developments in the technology of magnetic resonance image acquisition systems have opened interesting opportunities for the study of shoulder pathologies as well as for the assessment of the rehabilitation process. Specifically, the open-field architectures of MR systems with horizontal and vertical access allow for the study of the shoulder complex in different key positions during arm flexion and abduction. Whereas the study of the sub-acromial space deformations from $3 \mathrm{D}$ reconstructed structures has recently provided promising results in [2][3] only static information was processed for the extraction of relevant features.

Motion is an essential cue in the painful shoulder diagnosis. Most of the previous research about dynamic shoulder modelling is based on kynematic constraints [4] [5] [7] and therefore, it is computationally expensive. Furthermore, the variation of interstructural distances observed in MR imaging is difficult to integrate in kynematic models. This paper proposes a new image-based modelling approach for the analysis of the sub-acromial space deformation during arm flexion and abduction.

The organization of the rest of the paper is as follows. Section 2 presents the segmentation of MR image sequences, followed by the 3D reconstruction approach. Section 3 shows and validates our reconstruction results, while section 4 describes the extraction of relevant features for describing shoulder motion. Finally, we draw the conclusions and describe future work.

\section{Segmentation and 3D reconstruction}

\subsection{Semi-automatic segmentation approach}

Prior to the $3 \mathrm{D}$ reconstruction of the anatomical structures from $2 \mathrm{D}$ image sequences, a segmentation step is necessary to identify the regions of interest corresponding to the bony structures. Our approach is task-oriented and well-adapted to particular aspects of MR shoulder images such as: a) The morphology and global appearance of the bony structures varies significantly in different planes. The bony structures are not textured, and their appearance is dark and homogeneous in T1-weighted MR images. b) The structure-background transition is rather smooth, which results in blurred boundaries of the regions of interest in the image. c) The average brightness exhibits a significant inter-slice variance during the same MR sequence. Due to this variability and to the large number of images contained in one MR sequence, standard segmentation approaches based on user-specified thresholds are not recommendable; d) The complex morphology of the bony structures involved in the musculo-skeletal shoulder complex prevents from performing 3D segmentation, since the number of compact regions may vary from one slice to the next.

The database contains three types of parallel planar image sequences corresponding to three different orientations of the reference plane : saggital, axial and coronal respec- 
tively (see Fig. 1a). Due to the significant length of the image sequences, a minimal user intervention in the segmentation process is desirable. A user-friendly interface is designed for the selection of a rectangular region of interest (ROI) in the image. This interface allows the user to scan the entire sequence at the desired speed, to pause for a more thorough image examination and finally to draw a rectangular region framing the structures of interest throughout the entire sequence. The ROI is drawn only on the first image of the currently analyzed sequence and automatically mapped afterwards on every subsequent image belonging to the same sequence.
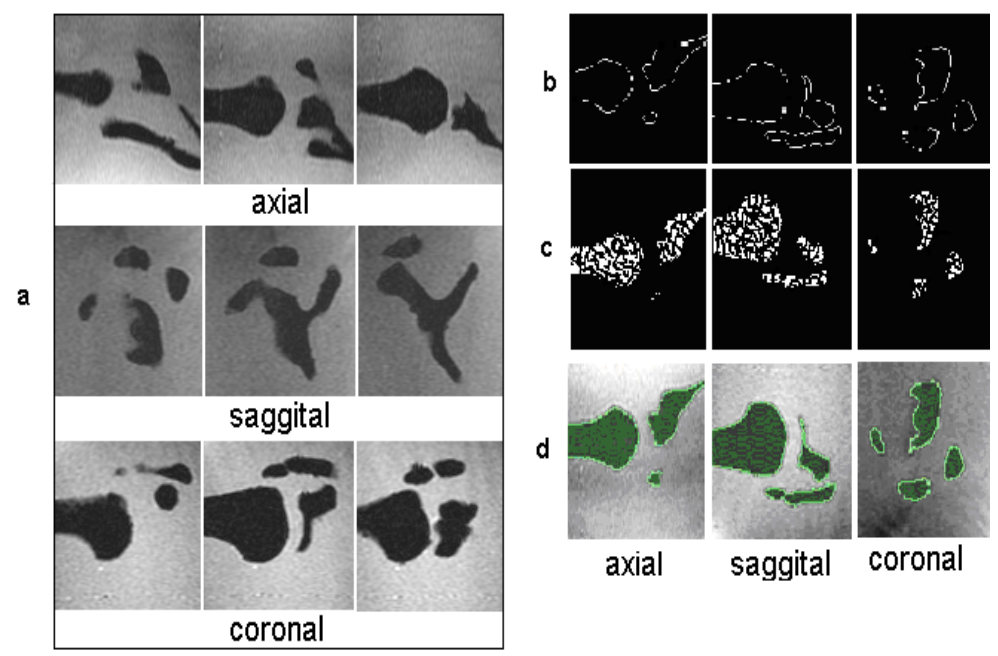

Figure 1. a) relevant samples of MR shoulder images corresponding to three standard orthogonal views b) edge image; c) seed regions for pixel aggregation; d) final segmentation result.

Our segmentation method combines edge detection and region growing. First, the gradient image is computed with Sobel convolution masks. The magnitude of the gradient conveys local information about the strength of the edges. While lowmagnitude edges correspond usually to noise, high-magnitude edges are more likely to correspond, at least partially, to the contours of the bony structures. High-magnitude edges are selected with respect to a threshold representing the average value of the gradient magnitude over the image. The contours in the thresholded image are not closed (see Fig.1b), due to local disconnections generated by the partial volume effect. Thus, a second segmentation step is necessary. This step consists in iterative pixel aggregation and uses the contour information to specify the similarity measure for candidate inclusion. Seed specification is automatic and histogram-based. Considering the global intensity histogram of the ROI, the seeds are defined as pixels with intensity values belonging to the 5\% inferior range of the histogram. As shown in Fig. 1c, most of the seed pixels are distributed in small-sized, compact regions. Therefore, a labelling of compact seed regions is performed first.

The similarity measure is computed with respect to the edge information and updated at each iteration. For every labelled growing region $R_{n}$, a corresponding list $L_{n}$ is cre- 
ated. This list $L_{n}$ contains contour pixels belonging to the labelled region $R_{n}$ as well as non-contour pixels adjacent to $R_{n}$ and to at least one contour pixel. After sorting the list in an ascending order, the similarity threshold set to the median value in the list. This similarity measure is consistent with the intensity variation inside the bony structures.

At each iteration, adjacent pixels to a growing region are aggregated if their intensity exceeds the similarity threshold. While parallel pixel aggregations are performed for every labeled region, region merging is allowed after each iteration. Finally, we obtain a pairwise correspondence between the bony structures present in the current image and the final regions resulting from segmentation and merging (see Fig. 1d). The parallel region growing process stops when no candidate for inclusion in any of the regions satisfies the similarity measure.

\subsection{D reconstruction}

We propose a 3D reconstruction approach using shape-based interpolation and extrapolation (see Fig. 2). Our interpolation technique insures a smooth transition between every two adjacent input shapes and is based on morphological morphing and "splitting". After interpolation, a closing surface step is performed using a new contour-based extrapolation technique. While other morphing techniques for shape interpolation have been previously proposed [8], our approach deals with anatomical structures with complex geometry, allowing a coherent integration of the "closing" and "morphing" sequences, and an adjustable uniform inter-slice resolution as well. Figure 2 shows the diagram of the proposed 3D reconstruction algorithm. After rendering, a size-preserving surface fairing approach is implemented as a slightly different version from [9].

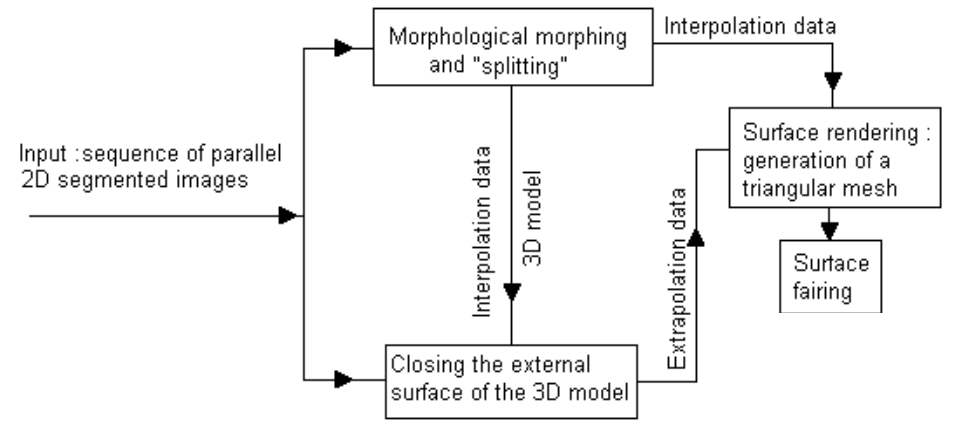

Figure 2. Diagram of the proposed 3D reconstruction algorithm

Morphological morphing of two simple compact regions

This morphing technique generates a gradual transition between two compact and partially overlapping shapes, as shown in Fig. 3. The mathematical formalism of this approach is described in [6]. While this simple technique works well for compact 
objects, the bony structures of the shoulder complex exhibit a complex geometry, and therefore, 'branching' problems may occur.

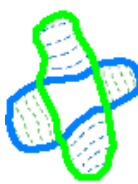

a)

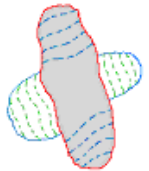

b)

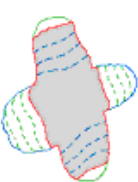

c)

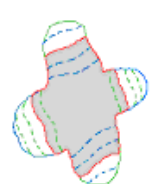

d)

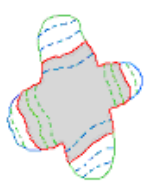

e)

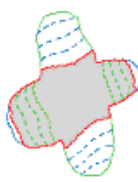

f)

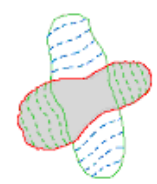

g)

Figure 3. Morphing algorithm : a) superposition of the initial shape $\operatorname{obj}_{1}$ (green boundary) and the final shape $o b j_{2}$ (blue boundary); the iterative conditional dilations of $\mathrm{obj}_{0}=\mathrm{obj}_{1} \cap \mathrm{obj}_{2}$ with respect to $\mathrm{obj}_{1}$ and of $\mathrm{obj}_{2}$ are in dashed lines; b)initial shape $\left.\left.\left.\left.\left(\mathrm{obj}_{1}\right) ; \mathrm{c}\right), \mathrm{d}\right), \mathrm{e}\right), \mathrm{f}\right)$ morphing iterations : intermediate objects are grey with red boundaries; g) final shape $\left(\mathrm{obj}_{2}\right)$

A morphological "splitting" approach

A "branching" situation as shown in Fig. 4 occurs when two disjoint regions in slice $i$ correspond to the same compact region in slice $i+1$. We give a particular attention to the "branching" problem, since it has important repercussions on interpolation as well as on surface rendering processes.

The idea is to divide the region resulting from "branch merging" into a sub-set of regions in order to allow a one-to-one correspondence. While the traditional solution to this problem is based on the Voronoi region diagram, we propose a more natural morphological "splitting" approach. For simplicity, we consider the situation when two binary, disjoint, and compact objects $o b j_{11}$ and $o b j_{12}$ in slice 1 merge into one compact object $o b j_{2}$ in adjacent slice 2. The "splitting" approach is to separate $o b j_{2}$ into $o b j_{21}$ and $o b j_{22}$ as shown in Fig. $4 \mathrm{~d}$.

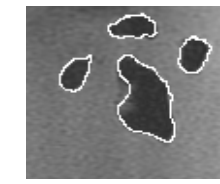

a) MR slice $i$

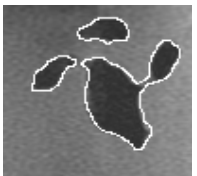

b) MR slice $i+1$

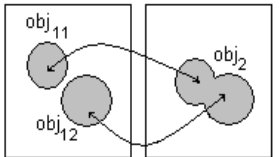

c)

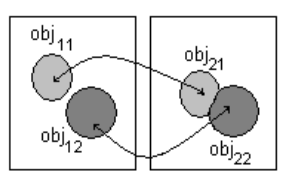

d)

Figure 4. "Branching" in a shoulder MR sequence : a) three disjoint scapula(S) regions; b) merging of the previous right-most two scapula regions into one region. S-scapula; C-clavicle; c) inter-slice region correspondence before splitting; inter-slice region correspondence after splitting;

The main steps of our "splitting" approach are :

Step 1. Perform simultaneous and iterative non-conditional dilations on $o b j_{11}$ and $o b j_{12}$ obtaining $o b j d_{11}$ and $o b j d_{12}$ respectively, until $o b j_{2} \subseteq\left(o b j d_{11} \cup o b j d_{12}\right)$. Since $o b j_{11}$ and $o b j_{12}$ are disjoint regions, and $o b j_{2}$ is a compact region, it can be easily proven that $\left(\operatorname{objd}_{11} \cap \operatorname{objd}_{12}\right) \neq \Phi$. The morphological dilation operator is shape preserving, thus $o b j d_{11}$ and $o b j d_{12}$ preserve the shape of $o b j_{11}$ and $o b j_{12}$ respectively.

Step 2. Apply the watershed transform [12] to $o b j_{2}$ and extract the watershed line wshl (i.e. the separation frontier between the regions detected with the watershed trans- 
form). As a result of "branch merging", $o b j_{2}$ preserves to a given extent the shape of both initial "branches", $o b j_{11}$ and $o b j_{12}$, thus the watershed transform is to generate the inter-slice one-to-one region correspondence. However, over-segmentation occurs very often when applying the watershed transform.

Step 3. Extract the separation line sep that divides $o b j_{2}$ in $o b j_{21}$ and $o b j_{22}$ :

sep $=w \operatorname{shl} \cap\left(\right.$ objd $_{11} \cap$ objd $\left._{22}\right)$

This step eliminates the over-segmentation effect. After the minimum number of iterative dilations performed at step 1, the separation limit between $o b j_{21}$ and $o b j_{22}$ is to be found in the overlapping region of objd $_{11}$ and $\operatorname{objd}_{12}$.
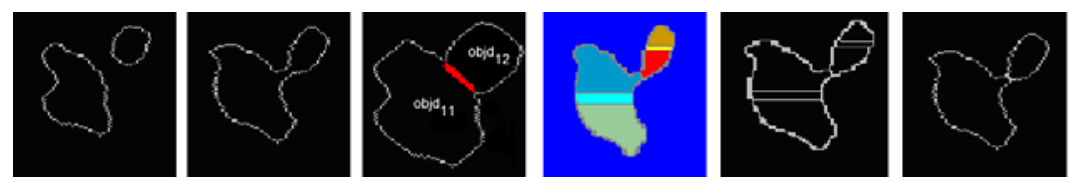

Figure 5. The "splitting" approach : a) two "branches", $o b j_{11}$ and $o b j_{12}$; b) object $o b j_{2}$ resulting from the "merging" of the two "branches"; c) step 1 : objd 11 ○objd 22 is in red; d) step 2 : over-segmentation of $o b j_{2}$ in 6 classes (labeled with different colors) with the watershed transform ; e) step 2 : watershed line; f) step 3 : splitting $o b j_{2}$ into $o b j_{21}$ and $o b j_{22}$.

Fig. 5 illustrates the main steps in the proposed "splitting" approach. This technique may be easily extended to three or more compact and disjoint "branches" merging into one compact region.

After creating a one-to-one correspondence between regions in adjacent slices, interpolation is performed with the technique described in [6].

The surface closing step is implemented as a contour-based extrapolation, described in detail in [6]. This step is necessary to correct the appearance of a cut-off cylinder of the interpolated $3 \mathrm{D}$ model.

\section{Model Validation}

The database for this study contains T1-weighted MR shoulder sequences from three orthogonal views: axial, sagittal and coronal, and corresponding to 6 human healthy subjects. The average length of a sequence is of 30 images. The images are stored in an uncompressed format and contain 256 gray levels. The size of the images is $256 \times 256$ pixels, while the intra-slice pixel resolution is of $1.25 \mathrm{~mm}$. The value of slice thickness is set to $7 \mathrm{~mm}$, which is a reasonable trade-off between the strength of the partial volume effect and the temporal extent of the acquisition process.

The reconstructed 3D shoulder model is to reproduce with high accuracy the real human bony structures at a 1:1 scale. Since a reference model is not available, we first perform an internal validation of our model. Specifically, for every human shoulder we build three models using the saggital, coronal, and axial sequences respectively. Next, we compare the models in order to assess their degree of similarity. If the models are quasi-identical, then the proposed 3D reconstruction method yields robust and 
reliable results. Model comparison is implemented in Polyworks, a software developed by Innovmetric Inc. and dedicated to the inspection of 3D models.

Error was computed in the region of interest, i.e. the structures surrounding the subacromial space. As shown in Fig. 6a, a colour code allows us to visualize the spatial distribution of the error magnitude. Green and light blue encode low magnitude values for positive and negative error respectively. For the case shown in Fig. 6a, the saggital and axial models have a high similarity level, and thus are considered quasi-identical. Table 1 centralizes the information about the inter-model comparison.

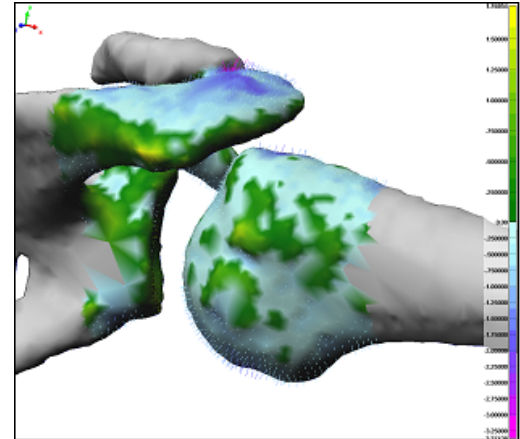

a)

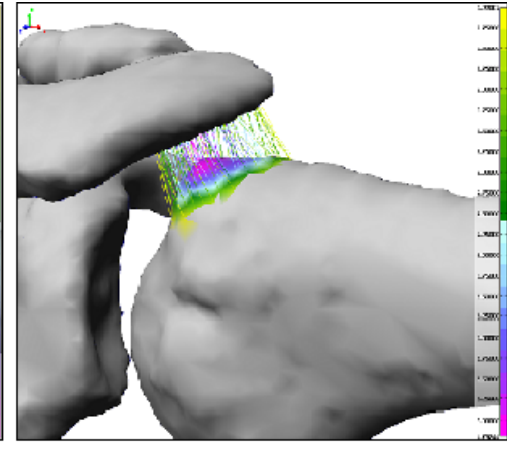

b)

Figure 6. Model validation in Polyworks: a) comparison of coronal and axial models corresponding to the same human shoulder b) computation of the acromio-humeral distance.

Internal validation results in low-valued average errors for every human shoulder in the database. Therefore, the 3D reconstructed models are considered to be reliable for the further computation of inter-structural distance measures.

To verify the 1:1 scale factor of the $3 \mathrm{D}$ reconstructed models, the acromio-humeral distance is computed as shown in Fig. 6b. The mean acromio-humeral distance for the shoulders in our database is 6.0151 , while the standard deviation is 0.659124 . These values are coherent with statistical anatomical measures for healthy subjects.

Table 1. Results obtained after comparing of the coronal and axial models shown in Fig 6a.

\begin{tabular}{|r|r|}
\hline \multicolumn{2}{|c|}{ Error distribution } \\
\hline Model \#1 & Axial \\
\hline Model\#2 & Coronal \\
\hline \#Points & 5345 \\
\hline Mean error & -0.130989 \\
\hline StdDev & 0.606965 \\
\hline Maximum positive error & 1.768526 \\
\hline Maximum negative error & -3.374344 \\
\hline Pts within $+/-(1 *$ StdDev $)$ & $3758(70.308700 \%)$ \\
\hline Pts within $+/-(2 *$ StdDev $)$ & $5118(95.753040 \%)$ \\
\hline Pts within $+/-(3 *$ StdDev $)$ & $5317(99.476146 \%)$ \\
\hline
\end{tabular}




\section{Feature extraction}

For the human shoulders in the database the acquisition process was performed at several key positions during arm elevation and abduction. Next, the acromio-humeral distance was computed. Fig. 7 illustrates the variation of the acromio-humeral distance with respect to the angular position during arm flexion and abduction.

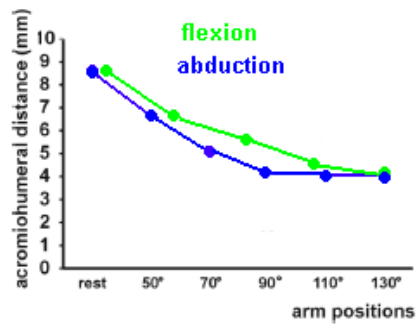

Figure 7. The variation of the average acromio-humeral distance for healthy human subjects during arm flexion and abduction

The results show that the $3 \mathrm{D}$ acromio-humeral distance is more reliable than the corresponding 2D equivalent, measured manually by physical therapists on a graphical interface embedded in the acquisition system [2].

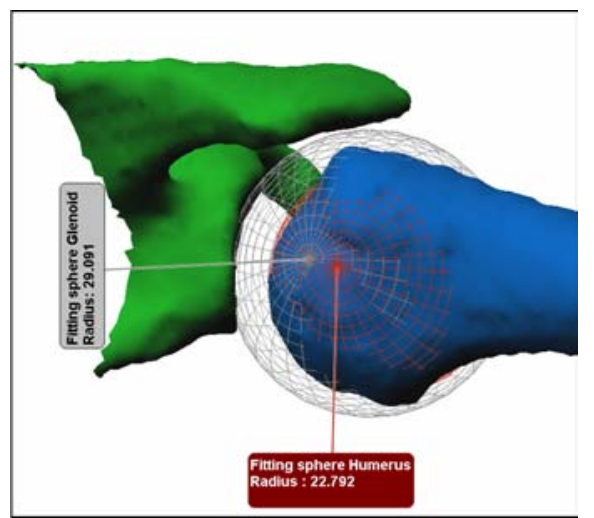

Figure 8. Relative position of spheres matched on the humeral head and on the glenoid at rest.

Moreover, since the glenoid cavity and the humeral head are in relative rotation during arm movements, we propose a new descriptor for assessing the surface compatibility of the two structures during motion. In fact, if the convexity of the humeral head does not match the concavity of the glenoid cavity, or if their rotation is not concentric, then the sub-acromial space is reduced under a critical value, resulting in pressure on ligaments and tendons and in an increased risk of injury. Therefore, spheres are matched on each of the rotating surfaces. The matching algorithm minimizes the distance between the spherical surface and the reconstructed anatomical surface over the 
region of interest. Results are shown in Fig. 8. For the healthy human shoulders in our database, the two spheres were almost concentric (average distance between centres was $2.178 \mathrm{~mm}$ ), and the distance between the spherical centres was conserved during arm motion. Moreover, the average difference between the spherical radii $(5.8 \mathrm{~cm})$ represents a simple descriptor of the geometry of the subacromial space. Future work will focus in assessing the reliability of spherical motion description for the diagnosis of shoulder pathologies.

\section{Conclusions and future work}

This paper presents a new end-to-end modeling technique for the study of the shoulder complex in motion. The input data consists of MR sequences acquired in three orthogonal planes and corresponding to key positions during arm flexion and abduction. The output data contains 3D models of the shoulder complex in motion as well as descriptors of the subacromial space deformation. The proposed modeling approach consists of three main modules: segmentation, 3D reconstruction, and feature extraction. The segmentation module integrates region and edge information in a coherent manner. Standard 3D reconstruction algorithms do not perform well on our input data, since the scanned volume is sampled in $7 \mathrm{~mm}$-thick slices. Thus, we propose a new reconstruction technique based on morphological morphing between adjacent slices and on contour-based extrapolation of extreme slices. Model validation is difficult, since no direct access is possible to in vivo human bony structures of the shoulder complex. We implement an internal validation approach, based on comparing the three models built for the same human shoulder. Next, descriptors of the subacromial space deformation during arm motion are computed. The discrete temporal variation of the AHD distance is consistent with previous studies [2]. Moreover, we propose a descriptor for assessing the compatibility of two complementary surfaces, one concave and the other convex, during relative rotation.

While our modeling approach is task-oriented, it is easy to adapt it for the study of other joints, such as elbow, knee and ankle. Thus, we provide a simple, yet efficient method for modeling complex musculo-skeletal structures in motion.

Future work will focus on modeling motion as a continuous process. While MR acquisition will be performed only at discrete moments, temporal interpolation and view morphing [10] will allow for the continuous modeling of arm flexion and abduction. In addition, descriptors for assessing surface convexity and concavity, as well as for measuring local surface irregularities [11] will be tested for implementation.

\section{Acknowledgements}

This research was supported by a grant from Fonds de la Recherché en SantéQuébec. Innovmetric Software Inc. has kindly provided one license for Polyworks. 


\section{References}

1. van der Heijden GJ: Shoulder disorders: a state of the art review. Baillieres Best Pract Res Clin Rheumatol, 13(2), 1999,287-309

2. Hébert, L.J., Moffet, H., Dufour, M., and Moisan,C.: Acromiohumeral distance in a seated position in persons with impingement syndrome. Journal of Magnetic Resonance Imaging, vol. 18( 1), 2003, $72-79$

3. Graichen H, Bonel H, Stammberger T, et al. : Three-dimensional analysis of the width of the subacromial space in healthy subjects and patients with impingement syndrome, AJR Am J Roentgenol 172 (1999), 1081-1086.

4. Engin, A. E., and Tumer, S. T.:Three-Dimensional Kinematic Modelling of the Human Shoulder Complex - Part I: Physical Model and Determination of Joint Sinus Cones", ASME Journal of Biomechanical Engineering, Vol. 111, 1989, 107-112.

5. Tumer, S. T. and Engin, A. E.: Three-Dimensional Kinematic Modelling of the Human Shoulder Complex - Part II: Mathematical Modelling and Solution via Optimization, ASME Journal of Biomechanical Engineering, Vol. 111, 1989, 113-121

6. Branzan-Albu, A., Schwartz, JM, Moisan, C., and Laurendeau, D.: Integrating geometric and biomechanical models of a liver tumour for cryosurgery simulation. Proc. of Surgery Simulation and soft Tissue Modeling IS4TM 2003, 121-131

7. Maurel, W. and Thalmann, D.: Human shoulder modelling including scapulo-thoracic constraint and joint sinus cones. Computer\&Graphics, New York, vol.24(2), 1998, 203-218

8. Bors, A., Kechagias, L., and Pitas, I.: Binary morphological shape-based interpolation applied to 3-D tooth reconstruction. IEEE Trans. Med. Imag., vol.21, Feb. 2002, 100-108

9. G.Taubin, "A signal processing approach to fair surface design”, Computer Graphics, vol.29, 1995, 351-358.

10. Manning, A. and Dyer, R.C.: Interpolating view and scene motion by dynamic view morphing. In Proc. of the IEEE Int. Conf. on Computer Vision and Pattern Recognition (CVPR'99), 1999, 388-394.

11. Yushkevich, P. , Pizer, S.M., Joshi, S., and Marron, J.S.: Intuitive, Localized Analysis of Shape Variability. International Conference on Information Processing in Medical Imaging, 2001, 402-408

12. Serra J.: Image Analysis and Mathematical Morphology. New York : Academic, 1982. 